\title{
Investigation of Synchronization for Social Network with Local Bridge via Coupled Rulkov Maps
}

\author{
Tomoya Shima $\uparrow$, Yoko Uwate $\dagger$, Thomas Ott $\$$ and Yoshifumi Nishio $\dagger$ \\ $\dagger$ Dept. of Electrical and Electronic Engineering, Tokushima University, \\ 2-1 Minami-Josanjima, Tokushima, 770-8506, Japan \\ phone:+81-88-656-7470, fax: +81-88-656-7471 \\ Email:\{s-tomoya, uwate, nishio\}@ee.tokushima-u.ac.jp \\ $\$$ Institute of Applied Simulation, Zurich University of Applied Sciences, \\ Einsiedlerstrasse 31a, 8820 Waedenswil, Switzerland \\ phone:+41-058-934-56-84 \\ Email:thomas.ott@ zhaw.ch
}

\begin{abstract}
It is important to understand dynamics of social network. We focus on "local bridge" in the social network. In this study, we investigate influence of the local bridge in the social network via synchronization phenomena of Rulkov maps applying for coupled maps. From simulation results, in the social network with local bridge, the propagation wave switches between full synchronization and clustering by the local bridge.
\end{abstract}

\section{INTRODUCTION}

Understanding dynamics of social network is important because "social network analysis" has been used not only for analyzing modern society but also for physics, biology and information science by many researchers [1]-[4]. The social network is structure which shows social relation, e.g. aerial line, infection with a virus, World Wide Web and so on.

In this study, we focus on "local bridge" which is found by M. Granovetter [5] and is one of phenomena in social network. We need to explain keyword "bridge" before explaining the local bridge. The bridge is an path in network which provides the only route between two points. Example of the bridge is shown in Fig. 1.

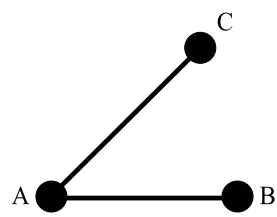

Fig. 1. Example of the bridge.

Here, the bridge between $\mathrm{A}$ and $\mathrm{B}$ provides the only route which can flow information or influence from any contact of A to any contact of $\mathrm{B}$. The function of the bridge is called "bridging function". The bridge path happens only rarely in large network actually. However, the bridging function may be provided locally. Its phenomenon shows in Fig. 2.

In Fig. 2, the shortest route from 1 to 25 is $1-25$. One of second shortest route from 1 to 25 is 1-4-5-8-9-11-13-

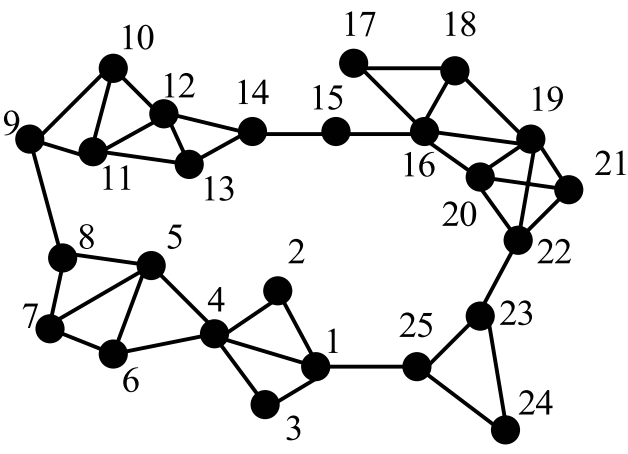

Fig. 2. Example of the local bridge.

14-15-16-20-22-23-25. The 1-25 route is not strictly bridge because other routes construct. However, the 1-25 route is predominantly shorter than other routes. In this case, the bridging function is provided locally by the 1-25 route and this bridge is called the local bridge. Similarly, it is found that $8-9,14-15,15-16$ and $22-23$ route are local bridge. In the social network, the local bridge affects the entire network in terms of propagation of information. Because the local bridge creates more and shorter routes.

In order to analyze complex phenomena of the social network with local bridge, we use coupled maps [7]. Generally, the coupled maps is used as general models for the complex dynamics, e.g. biological systems, economic activities and neural network. Coupled oscillatory systems can also produce interesting phase patterns, "clustering" and complex phase patterns. Moreover, a discrete map for spiking-bursting neural behavior was proposed by Rulkov [6]. Rulkov maps can be useful for understanding the dynamical mechanism of oscillators in "the large scale network". In this study, we use Rulkov maps applying for the coupled maps. Because the social network has features of cluster and the large scale network, and we consider that the propagation of information 
on the social network is realized by the Rulkov maps applying for the coupled maps.

In this study, we show synchronization of the social network of Rulkov maps if a coupling strength $g$ and a control parameter $\alpha$ are changed. Thereby, we observe the influence of the local bridge on the social network.

\section{Two coupled RulKov MaPs}

In this section, we observe fundamental synchronization for two coupled Rulkov maps if the coupling strength $g$ and the control parameter $\alpha$ are changed in order to compare synchronization for the social network of Rulkov maps which is large scale network of two coupled Rulkov maps. The equation of the Rulkov maps is shown as follows.

$$
\begin{aligned}
x_{i, n+1} & =f\left(x_{i, n}, y_{i, n}+\beta_{i, n}\right), \\
y_{i, n+1} & =y_{i, n}-\mu\left(x_{i, n}+1\right)+\mu \sigma_{i}+\mu \sigma_{i, n},
\end{aligned}
$$

$f\left(x_{n}, y\right)=\left\{\begin{array}{lc}\alpha /\left(1-x_{n}\right)+y, & x_{n} \leq 0 \\ \alpha+y, & 0<x_{n}<\alpha+y \text { and } x_{n-1} \leq 0 \\ -1, & x_{n} \geq \alpha+y \text { or } x_{n-1}>0\end{array}\right.$

where $x$ and $y$ are the fast and slow dynamical variables, $\alpha$ is a control parameter of the map. The coupling between two cells is provided by the current flowing from one cell to the other. This coupling is modeled by

$$
\begin{aligned}
\beta_{i, n} & =g_{j i} \beta^{e}\left(x_{j, n}-x_{i, n}\right), \\
\sigma_{i, n} & =g_{j i} \sigma^{e}\left(x_{j, n}-x_{i, n}\right),
\end{aligned}
$$

where $g$ denotes the coupling strength. In the numerical simulations the values of the coefficients are set to be equal: $\beta^{e}=1.0$ and $\sigma^{e}=1.0$. The other parameters has the following values: $\mu=0.001$, and $\sigma=0.15$. The coupling between the maps is symmetrical, i.e., $g_{j i}=g_{i j}=g$.

First, we investigate the boundary of synchronization and asynchronization for two coupled Rulkov maps if the difference between the control parameter $\alpha$ of one cell and the other increases, or the coupling strength $g$ is changed. By this investigation, Here, $\alpha_{1}$ of one cell is fixed at 5.0, $\alpha_{2}$ of the other cell is $\alpha_{1}+\Delta \alpha$. Two bursting waves are synchronized at the in-phase as shown in Fig. 3. And Fig. 4 is shown asynchronization. The boundary of synchronization and asynchronization for two coupled Rulkov maps is shown in Fig 5. From this result, if $g$ or $\Delta \alpha$ is getting larger, two coupled Rulkov maps tends to synchronize. Also, if $g$ is larger than 0.05 , two coupled Rulkov maps has highly synchronous.

\section{Social NETWORK OF RULKov MAPS}

We consider the case of the social network. In this study, this social network is shown in Fig. 2. In order to simulate on the social network, we write an equation based on a chain of

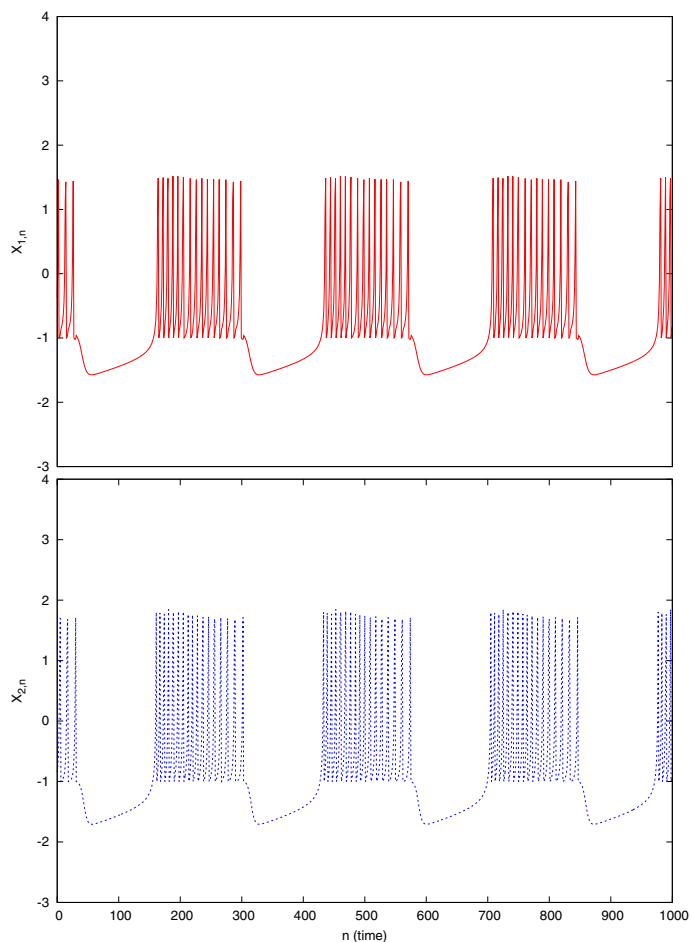

Fig. 3. In phase synchronization $\left(\alpha_{1}=5.0, \alpha_{2}=5.5, g=0.03\right)$.

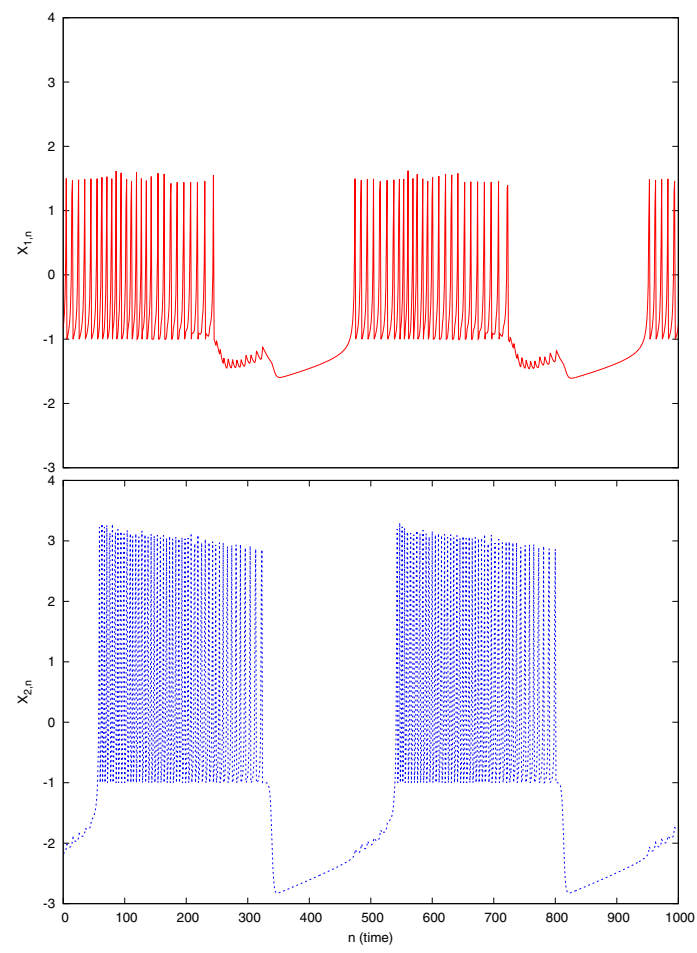

Fig. 4. Asynchronization $\left(\alpha_{1}=5.0, \alpha_{2}=7.9, g=0.03\right)$. 


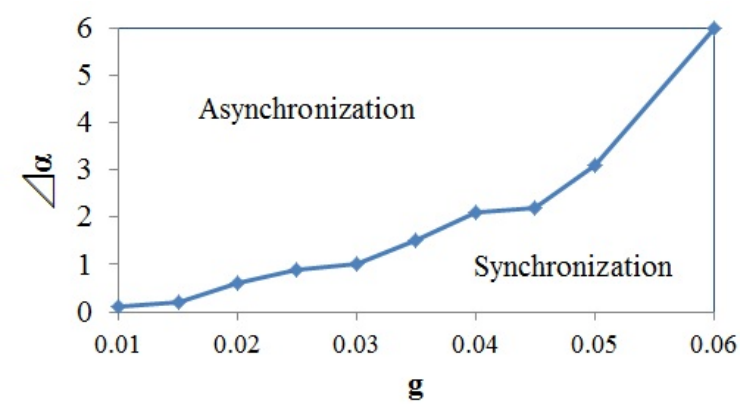

Fig. 5. Boundary of synchronization and asynchronization.

coupled maps [7].

$$
\begin{aligned}
& x_{i, n+1}=f\left(x_{i, n}, x_{i, n-1}, y_{i, n}\right)+\frac{g}{N} \sum_{j \in C_{j}}\left(x_{j, n}-x_{i, n}\right), \\
& y_{i, n+1}=y_{i, n}-\mu\left(x_{i, n}+1\right)+\mu \sigma_{i}+\mu \frac{g}{N} \sum_{j \in C_{j}}\left(x_{j, n}-x_{i, n}\right), \\
& i=1, \ldots, N,
\end{aligned}
$$

where $x$ and $y$ are the fast and slow dynamical variables, $g$ is the coupling strength, $N$ is the number of cells in the social network, $\mu=0.001, \sigma=0.15, C_{j}$ is set of nodes which are connected to node $i$. The function $f()$ has the following form:

$$
f\left(x_{n}, y_{n}\right)=\left\{\begin{array}{l}
\alpha /\left(1-x_{n}\right)+y_{n}, \quad x_{n} \leq 0 \\
\alpha+y_{n}, \quad 0<x_{n}<\alpha+y_{n} \text { and } x_{n-1} \leq 0 \\
-1, \quad x_{n} \geq \alpha+y_{n} \text { or } x_{n-1}>0,
\end{array}\right.
$$

Similarly, we investigate the boundary of synchronization and asynchronization for the social network of Rulkov maps if the difference between the control parameter $\alpha$ of each cell in the social network increases, or the coupling strength $g$ is changed. Here, we determine $\alpha_{0}$ which is standard value and is 5.0. $\alpha$ of each cell is $\alpha_{0}+\Delta \alpha . \Delta \alpha$ is set for randomly distributed in the interval $[0: \Delta \alpha]$. The boundary of synchronization and asynchronization for the social network of Rulkov maps is shown in Fig. 6. From this result, the social network of Rulkov maps tends to lowly have synchronous. Also, if $g$ is larger than 0.07 , the social network of Rulkov maps has highly synchronous like two coupled Rulkov maps.

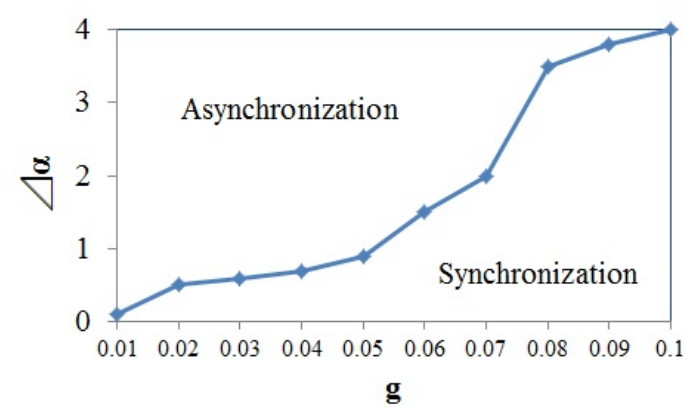

Fig. 6. Boundary of synchronization and asynchronization.
Next, we show synchronization of the social network of Rulkov maps which produce spiking-bursting behavior. In this study, we define two maps as synchronization if timing of bursting wave between two maps is right. Here, "full synchronization" is that all maps are synchronized at the inphase, and "clustering" is that maps is synchronized at the in-phase locally. The simulation results are shown Fig. 7. The horizontal axis is iteration time $n$ and the vertical axis is space $i$. The complex propagation wave can be observed when $g$ is 0.05 and $\Delta \alpha_{\text {max }}$ is 1.2 (see. Figs. 6 (a) and (b)). From Fig. 6 (a), we assume that the maps tend to converge full synchronization by repeating update. However, in the case of Fig. 6 (b), the maps switch between full synchronization and clustering. Also, when the maps switch from full synchronization to clustering, phase deviation occurs from the local bridges. In the case of Figs. 6 (a) and (b), the phase deviation occurs from local bridge 8-9 and 14-15. For Figs. 7 (c) and (d), we set $g=0.04$ and $\Delta \alpha_{\max }=1.0$. For Figs. 7 (e) and (f), we set $g=0.03$ and $\Delta \alpha_{\max }=0.7$. Similarly, for Figs. 7 (c) and (d), the phase deviation occurs from local bridge 8-9, for Figs. 7 (e) and (f), the phase deviation occurs from local bridge 14-15 and 22-23. From these result, in the social network with local bridge, the social network of Rulkov maps does not converges full synchronization. Because the maps switch between full synchronization and clustering by the local bridge.

\section{Conclusion}

In this study, we have studied influence of the local bridge in the social network via synchronization phenomena of Rulkov maps applying for the coupled maps. From simulation results, in the social bridge with local bridge, the propagation wave switches between synchronization and asynchronization by the local bridge. In the future works, we investigate synchronization phenomena of another social network which has larger scale.

\section{AcKNOWLEDGMENT}

This work was partly supported by JSPS Grant-in-Aid for Young Scientists 23700269.

\section{REFERENCES}

[1] S. Milgram, "The Small World Problem," Psychology Today, vol. 2, pp. 60-67, 1967.

[2] D. Watts and S. Strogatz, "Collective Dynamics of small-world' networks," Nature, vol. 393, pp. 440-442, 1998.

[3] A.L. Barabsi and R. Albert, "Emergence of scaling in random networks," Science, vol. 286, pp. 509-512, 1999.

[4] D. Watts, "Small worlds : the dynamics of networks between order and randomness," Princeton University Press, 2003.

[5] S. Mark, "The Strength of Weak Ties", Amerian Journal of Sociology, vol 78, pp 1360-1380, 1973.

[6] N.F. Rulkov, "Modeling of Spiking-Bursting Neural Behavior using Twodimensional Map,” Physical Rev., E, vol. 65, 041922, 2002.

[7] K. Kaneko, "Spatiotemporal Intermittency in Coupled Map Lattice," Prog. Theor. Phys, vol. 75, pp. 1033-1044, 1985. 


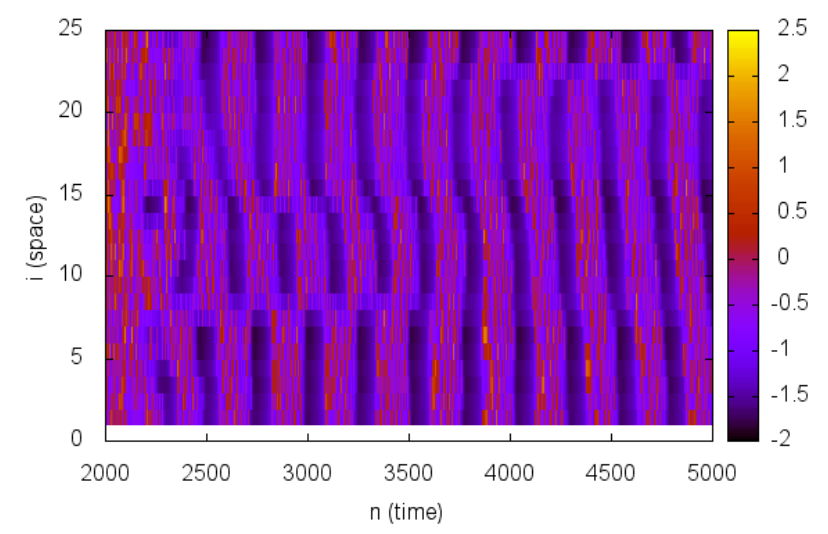

(a) $g=0.05, \Delta \alpha=1.2, n=2000-5000$.

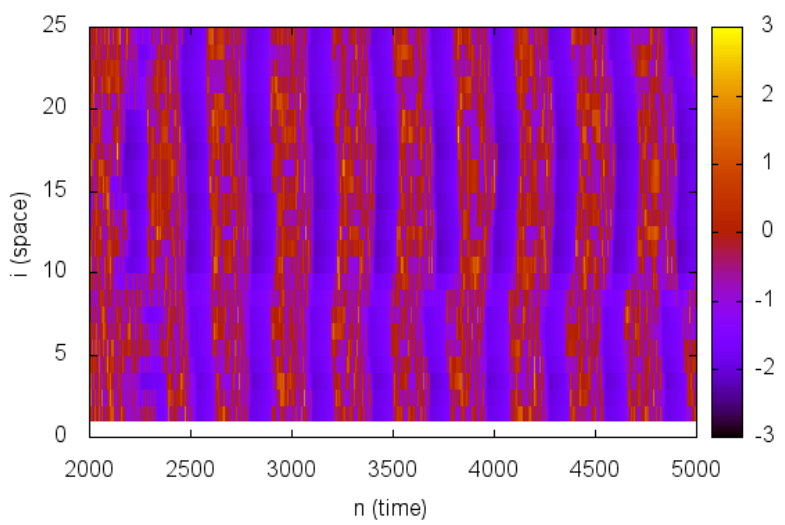

(c) $g=0.04, \Delta \alpha=1.0, n=2000-5000$.

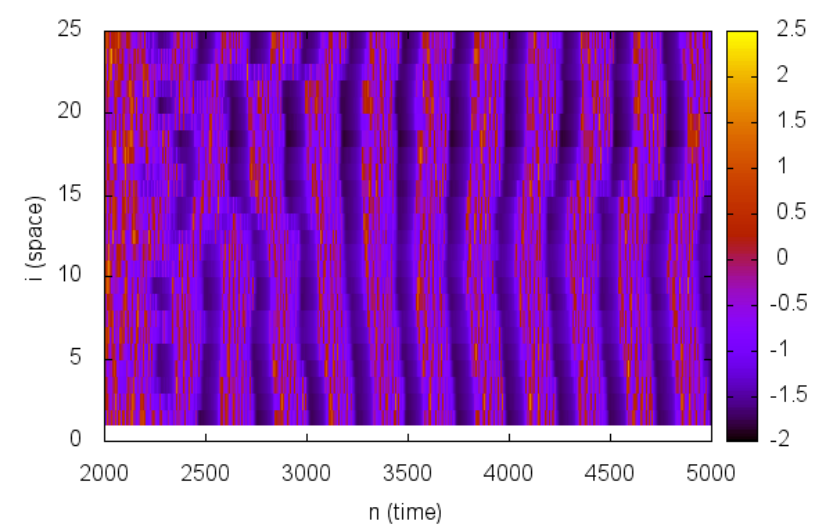

(e) $g=0.03, \Delta \alpha=0.7, n=2000-5000$.

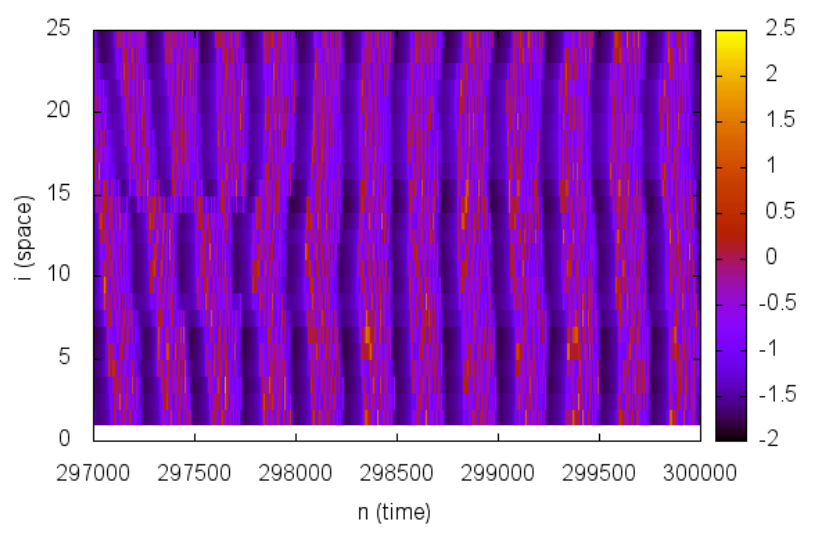

(b) $g=0.05, \Delta \alpha=1.2, n=297000-300000$.

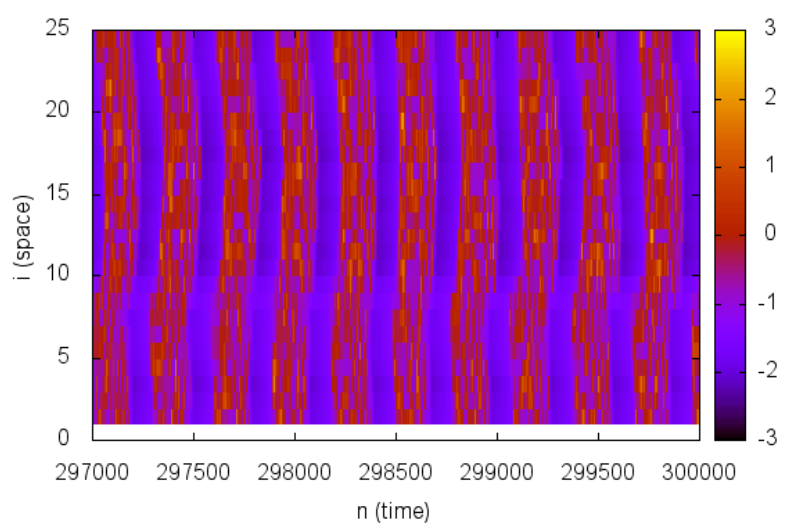

(d) $g=0.04, \Delta \alpha=1.0, n=297000-300000$.

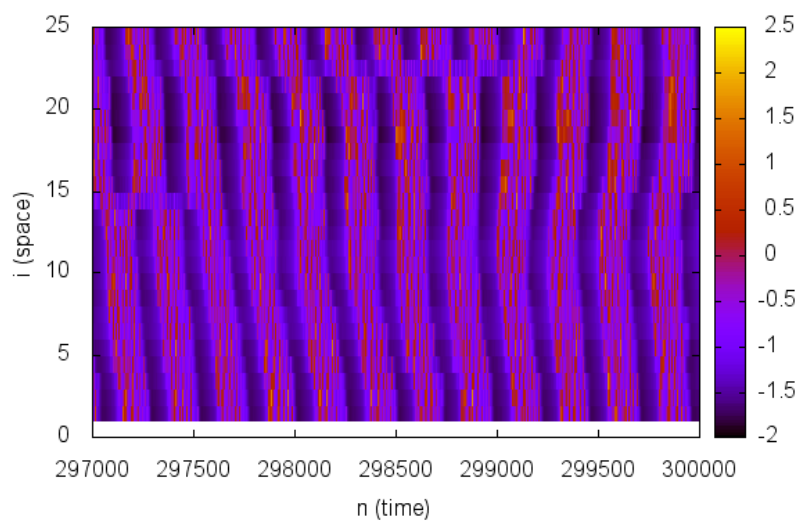

(f) $g=0.03, \Delta \alpha=0.7, n=297000-300000$.

Fig. 7. Propagation wave for the social network. 